\title{
Equivariant Join and Fusion of Noncommutative Algebras
}

\author{
Ludwik DABBROWSKI ${ }^{\dagger}$, Tom HADFIELD ${ }^{\ddagger}$ and Piotr M. HAJAC $\S$ \\ † SISSA (Scuola Internazionale Superiore di Studi Avanzati), \\ Via Bonomea 265, 34136 Trieste, Italy \\ E-mail: dabrow@sissa.it \\ $\ddagger$ G-Research, Whittington House, 19-30 Alfred Place, London WC1E 7EA, UK \\ E-mail: Thomas.Daniel.Hadfield@gmail.com \\ $\S$ Institytut Matematyczny, Polska Akademia Nauk, \\ ul. Śniadeckich 8, 00-656 Warszawa, Poland \\ E-mail: pmh@impan.pl \\ URL: http://www.impan.pl/ pmh/
}

Received June 30, 2015, in final form October 03, 2015; Published online October 13, 2015

http://dx.doi.org/10.3842/SIGMA.2015.082

\begin{abstract}
We translate the concept of the join of topological spaces to the language of $C^{*}$-algebras, replace the $C^{*}$-algebra of functions on the interval $[0,1]$ with evaluation maps at 0 and 1 by a unital $C^{*}$-algebra $C$ with appropriate two surjections, and introduce the notion of the fusion of unital $C^{*}$-algebras. An appropriate modification of this construction yields the fusion comodule algebra of a comodule algebra $P$ with the coacting Hopf algebra $H$. We prove that, if the comodule algebra $P$ is principal, then so is the fusion comodule algebra. When $C=C([0,1])$ and the two surjections are evaluation maps at 0 and 1 , this result is a noncommutative-algebraic incarnation of the fact that, for a compact Hausdorff principal $G$-bundle $X$, the diagonal action of $G$ on the join $X * G$ is free.
\end{abstract}

Key words: $C^{*}$-algebras; Hopf algebras; free actions

2010 Mathematics Subject Classification: 46L85; 58B32

\section{Introduction}

The join of topological spaces is a crucial concept in algebraic topology - it is used in the celebrated Milnor's construction of a universal principal bundle [9]. The goal of this paper is to provide a noncomutative-geometric version of the join of a Cartan compact Hausdorff principal bundle (no local triviality assumed) with its structure group. In particular, we obtain this way a noncommutative version of the diagonal $G$-action on the $n$-fold join $G * \cdots * G$ of a compact Hausdorff topological group $G$, which is the first step in Milnor's construction.

To make this paper self-contained and to establish notation and terminology, we begin by recalling the basics of classical joins, strong connections [6] and principal comodule algebras [7]. In the first section, we define the join and fusion $C^{*}$-algebra of an arbitrary pair of unital $C^{*}$-algebras. For commutative $C^{*}$-algebras our join construction recovers Milnor's definition in the case of compact Hausdorff topological spaces. In the second section, we deal with a join and fusion of a comodule algebra $P$ with the coacting Hopf algebra $H$. To define an $H$-comodule algebra structure that corresponds to the diagonal action in the classical setting, we provide a "gauged" algebraic version of the join and fusion $C^{*}$-algebras. (The gauged $C^{*}$-algebraic versions of the join and fusion are given respectively in [2] and [5].) The main result of this 
paper is Theorem 5.3 concluding the principality of the fusion comodule algebra of $P$ and $H$ from the principality of $P$.

\section{Classical join construction}

Let $I=[0,1]$ be the closed unit interval and let $X$ be a topological space. The unreduced suspension $\Sigma X$ of $X$ is the quotient of $I \times X$ by the equivalence relation $R_{S}$ generated by

$$
(0, x) \sim\left(0, x^{\prime}\right), \quad(1, x) \sim\left(1, x^{\prime}\right)
$$

Now take another topological space $Y$ and, on the space $I \times X \times Y$, consider the equivalence relation $R_{J}$ given by

$$
(0, x, y) \sim\left(0, x^{\prime}, y\right), \quad(1, x, y) \sim\left(1, x, y^{\prime}\right) .
$$

The quotient space $X * Y:=(I \times X \times Y) / R_{J}$ is called the join of $X$ and $Y$. It resembles the unreduced suspension of $X \times Y$, but with only $X$ collapsed at 0 , and only $Y$ collapsed at 1 . In particular, if $Y$ is a one-point space, the join $X * Y$ is the cone $C X$ of $X$. If $Y$ is a two-point space with discrete topology, then the join $X * Y$ is the unreduced suspension $\Sigma X$ of $X$.

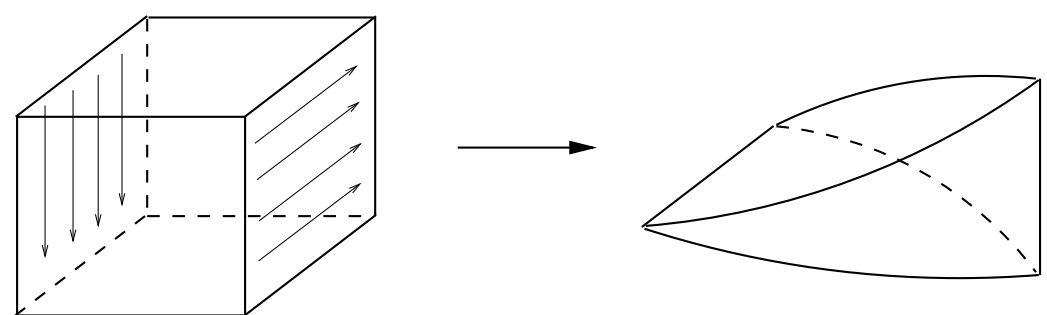

If $G$ is a topological group acting continuously on $X$ and $Y$ from the right, then the diagonal right $G$-action on $X \times Y$ induces a continuous action on the join $X * Y$. Indeed, the diagonal action of $G$ on $I \times X \times Y$ factorizes to the quotient, so that the formula

$$
([(t, x, y)], g) \longmapsto[(t, x g, y g)]
$$

makes $X * Y$ a right $G$-space. It is immediate that this continuous action is free if the $G$-actions on $X$ and $Y$ are free.

Consider $C X \times Y, X \times C Y$ and $X \times Y$ as $G$-spaces with the diagonal $G$-actions. Note that there is a continuous surjection $\pi_{I}: X * Y \ni[(t, x, y)] \mapsto t \in[0,1]$ such that $\pi_{I}^{-1}\left(\left[0, \frac{1}{2}\right]\right)$ and $\pi_{I}^{-1}\left(\left[\frac{1}{2}, 1\right]\right)$ are $G$-equivariantly homeomorphic to $C X \times Y$ and $X \times C Y$ respectively. Thus $X * Y$ is $G$-equivariantly homeomorphic to the gluing of $C X \times Y$ and $X \times C Y$ over $X \times Y$ :

$$
X * Y \cong(C X \times Y) \underset{X \times Y}{\sqcup}(X \times C Y) .
$$

If $Y=G$ with the right action assumed to be the group multiplication, we can construct the join $G$-space $X * Y$ in a different manner: at 0 we collapse $X \times G$ to $G$ as before, and at 1 we collapse $X \times G$ to $(X \times G) / R_{D}$ instead of $X$. Here $R_{D}$ is the equivalence relation generated by $(x, h) \sim\left(x^{\prime}, h^{\prime}\right)$, where $x h=x^{\prime} h^{\prime}$. More precisely, let $R_{J}^{\prime}$ be the equivalence relation on $I \times X \times G$ generated by

$$
(0, x, h) \sim\left(0, x^{\prime}, h\right) \quad \text { and } \quad(1, x, h) \sim\left(1, x^{\prime}, h^{\prime}\right), \quad \text { where } \quad x h=x^{\prime} h^{\prime} .
$$


The formula $[(t, x, h)] k:=[(t, x, h k)]$ defines a continuous right $G$-action on $(I \times X \times G) / R_{J}^{\prime}$. One can also easily check that the formula

$$
X * G \ni[(t, x, h)] \longmapsto\left[\left(t, x h^{-1}, h\right)\right] \in(I \times X \times G) / R_{J}^{\prime}
$$

yields a $G$-equivariant homeomorphism.

If we further specify also $X=G$ with the right action assumed to be the group multiplication, then the $G$-action on $X * Y=G * G$ is automatically free. Furthermore, since the action of $G$ on $X * G$ is free whenever it is free on $X$, we conclude that the natural action on the iterated join of $G$ with itself is also free. For instance, for $G=\mathbb{Z} / 2 \mathbb{Z}$ we obtain a $\mathbb{Z} / 2 \mathbb{Z}$-equivariant identification $(\mathbb{Z} / 2 \mathbb{Z})^{*(n+1)} \cong S^{n}$, where $S^{n}$ is the $n$-dimensional sphere with the antipodal action of $\mathbb{Z} / 2 \mathbb{Z}$.

\section{Strong connection and principal comodule algebra}

Let $H$ be a Hopf algebra with coproduct $\Delta$, counit $\varepsilon$ and bijective antipode $S$. Next, let $\delta: P \rightarrow P \otimes H$ be a coaction making $P$ a right $H$-comodule algebra. We shall frequently use the Heyneman-Sweedler notation (with the summation sign suppressed) for coproduct and coaction:

$$
\Delta(h)=: h_{(1)} \otimes h_{(2)}, \quad \delta(p)=: p_{(0)} \otimes p_{(1)} .
$$

Definition $3.1([7])$. Let $P$ be a right comodule algebra over a Hopf algebra $H$ with bijective antipode, and let

$$
B:=P^{\mathrm{co} H}:=\{p \in P \mid \delta(p)=p \otimes 1\} .
$$

be the coaction-invariant subalgebra. The comodule algebra $P$ is called principal if the following conditions are satisfied:

1) the coaction of $H$ is Hopf-Galois, that is, the map

$$
\operatorname{can}_{P}: P \underset{B}{\otimes} P \longrightarrow P \otimes H, \quad p \otimes q \longmapsto p q_{(0)} \otimes q_{(1)},
$$

(called the canonical map) is bijective,

2 ) the comodule algebra $P$ is right $H$-equivariantly projective as a left $B$-module, i.e., there exists a right $H$-colinear and left $B$-linear splitting of the multiplication map $B \otimes P \rightarrow P$.

Definition 3.2 ([3]). Let $H$ be a Hopf algebra with bijective antipode. A strong connection $\ell$ on a right $H$-comodule algebra $P$ is a unital linear map $\ell: H \rightarrow P \otimes P$ satisfying the following bicolinearity (i.e., left and right colinearity) and splitting conditions:

1) $(\mathrm{id} \otimes \delta) \circ \ell=(\ell \otimes \mathrm{id}) \circ \Delta,\left(\delta^{L} \otimes \mathrm{id}\right) \circ \ell=(\mathrm{id} \otimes \ell) \circ \Delta$, where $\delta^{L}:=\left(S^{-1} \otimes \mathrm{id}\right) \circ$ flip $\circ \delta$;

2) $\widetilde{\operatorname{can}_{P}} \circ \ell=1 \otimes \mathrm{id}$, where $\widetilde{\operatorname{can}_{P}}: P \otimes P \ni p \otimes q \mapsto(p \otimes 1) \delta(q) \in P \otimes H$.

We will use the Heyneman-Sweedler-type notation $\ell(h)=: \ell(h)^{\langle 1\rangle} \otimes \ell(h)^{\langle 2\rangle}$ with the summation sign suppressed. One can easily prove (see [7, p. 599] and references therein) that a comodule algebra is principal if and only if it admits a strong connection. Here we need the following slight generalization of this fact:

Lemma 3.3. Let $H$ be a Hopf algebra with bijective antipode. Then a right $H$-comodule algebra $P$ is principal if and only if it admits a (not necessarily unital) linear map $\ell: H \rightarrow P \otimes P$ satisfying the bicolinearity and splitting conditions of Definition 3.2. 
Proof. Assume that $\ell: H \rightarrow P \otimes P$ is a linear map satisfying the bicolinearity and splitting conditions. Let $\pi_{B}: P \otimes P \rightarrow P \otimes_{B} P$ be the canonical surjection. Define

$$
L: P \otimes H \ni p \otimes h \longmapsto \pi_{B}\left(p \ell(h)^{\langle 1\rangle} \otimes \ell(h)^{\langle 2\rangle}\right) \in P \underset{B}{\otimes} P .
$$

It follows immediately from the splitting property of $\ell$ that

$$
\operatorname{can}_{P}(L(p \otimes h))=p \widetilde{\operatorname{can}_{P}}(\ell(h))=p \otimes h .
$$

Applying id $\otimes \varepsilon$ to the splitting condition for $\ell$, we obtain $m \circ \ell=\varepsilon$, where $m$ is the multiplication map on $P$. Combining it with the left colinearity of $\ell$, which implies that

$$
\begin{aligned}
\delta\left(q_{(0)} \ell\left(q_{(1)}\right)^{\langle 1\rangle}\right) \otimes \ell\left(q_{(1)}\right)^{\langle 2\rangle} & =q_{(0)} \ell\left(q_{(3)}\right)^{\langle 1\rangle} \otimes q_{(1)} S\left(q_{(2)}\right) \otimes \ell\left(q_{(3)}\right)^{\langle 2\rangle} \\
& =q_{(0)} \ell\left(q_{(1)}\right)^{\langle 1\rangle} \otimes 1 \otimes \ell\left(q_{(1)}\right)^{\langle 2\rangle},
\end{aligned}
$$

we obtain

$$
\begin{aligned}
L\left(\operatorname{can}_{P}(p \otimes q)\right) & =\pi_{B}\left(p q_{(0)} \ell\left(q_{(1)}\right)^{\langle 1\rangle} \otimes \ell\left(q_{(1)}\right)^{\langle 2\rangle}\right)=\pi_{B}\left(p \otimes q_{(0)} \ell\left(q_{(1)}\right)^{\langle 1\rangle} \ell\left(q_{(1)}\right)^{\langle 2\rangle}\right) \\
& =\pi_{B}(p \otimes q) .
\end{aligned}
$$

Finally, the bicolinearity of $\ell$ implies that the formula $s(p):=p_{(0)} \ell\left(p_{(1)}\right)$ defines a left $B$-linear right $H$-colinear splitting of the multiplication map $B \otimes P \rightarrow P$. The reverse implication (principality $\Rightarrow$ existence of a bicolinear $\ell$ with the splitting property) follows from [3, Lemma 2.2].

\section{Join and fusion of unital $C^{*}$-algebras}

Let $\otimes_{\min }$ denote the spatial (minimal) tensor product. Due to the fact that minimal tensor products preserve injections (e.g., see [11, Proposition 4.22] or [12, Section 1.3]), for any unital $C^{*}$-algebras $A_{1}$ and $A_{2}$ the natural maps

$$
A_{1} \ni a \longmapsto a \otimes 1 \in A_{1} \underset{\min }{\otimes} A_{2} \quad \text { and } \quad A_{2} \ni a \longmapsto 1 \otimes a \in A_{1} \underset{\min }{\otimes} A_{2}
$$

are injective. This allows us to view $A_{1}$ and $A_{2}$ as subalgebras of $A_{1} \otimes_{\min } A_{2}$. We use this natural identification in what follows.

Definition 4.1. Let $A_{1}$ and $A_{2}$ be unital $C^{*}$-algebras, and let $C$ be a unital $C^{*}$-algebra equipped with two $C^{*}$-ideals $J_{1}$ and $J_{2}$ such that the direct sum $\pi_{1} \oplus \pi_{2}: C \rightarrow\left(C / J_{1}\right) \oplus\left(C / J_{2}\right)$ of the canonical surjections $\pi_{i}: C \rightarrow C / J_{i}, i \in\{1,2\}$, is surjective. We call the unital $C^{*}$-algebra

$$
A_{1} \underset{\pi}{\circledast} A_{2}:=\left\{x \in C \underset{\min }{\otimes} A_{1} \underset{\min }{\otimes} A_{2} \mid\left(\pi_{i} \otimes \mathrm{id}\right)(x) \in\left(C / J_{i}\right) \otimes_{\min } A_{i}, i \in\{1,2\}\right\}
$$

the fusion $C^{*}$-algebra of $A_{1}$ and $A_{2}$ over $C$. When $C$ is the $C^{*}$-algebra $C([0,1])$ of all continuous complex-valued functions on the unit interval and $\pi_{1}:=\mathrm{ev}_{1}$ and $\pi_{2}:=\mathrm{ev}_{0}$ are the evaluation maps respectively at 1 and 0 , we call $A_{1} \circledast_{\pi} A_{2}$ the join $C^{*}$-algebra of $A_{1}$ and $A_{2}$, and denote it by $A_{1} \circledast A_{2}$.

When $C$ is a commutative $C^{*}$-algebra, the projections $\pi_{i}$ correspond to inclusions of two disjoint closed subsets (playing the role of points 0 and 1) into a compact Hausdorff space (playing the role of $[0,1]$ ). Observe also that, if $A_{1}:=C(X)$ and $A_{2}:=C(Y)$ are the $C^{*}$-algebras of continuous functions on compact Hausdorff spaces $X$ and $Y$ respectively, then

$$
A_{1} \circledast A_{2}=C(X * Y) .
$$

On the noncommutative side, it is worth mentioning that the join of matrix algebras is used to construct the celebrated Jiang-Su $C^{*}$-algebra [8]. 


\section{Join and fusion of a principal $\boldsymbol{H}$-comodule algebra with $\boldsymbol{H}$}

Since a diagonal coaction is not in general an algebra homomorphism, to obtain an equivariant version of our noncommutative fusion and join constructions, we need to modify Definition 4.1 in the spirit of (2.1), (2.2). To avoid analytical complications that are tackled in [5], we also need to change the setting from $C^{*}$-algebraic to algebraic. In particular, we replace $\otimes_{\min }$ by $\otimes$.

Definition 5.1. Let $\delta: P \rightarrow P \otimes H$ be a right coaction making $P$ a comodule algebra, and let $C$ be a unital $C^{*}$-algebra equipped with two $C^{*}$-ideals $J_{1}$ and $J_{2}$ such that the direct sum

$$
\pi_{1} \oplus \pi_{2}: C \longrightarrow\left(C / J_{1}\right) \oplus\left(C / J_{2}\right)
$$

of the canonical surjections $\pi_{i}: C \rightarrow C / J_{i}, i \in\{1,2\}$, is surjective. We call the algebra

$$
\begin{aligned}
P \underset{\pi}{\circledast} H:= & \{x \in C \otimes P \otimes H \\
& \left.\left(\pi_{1} \otimes \mathrm{id}\right)(x) \in\left(C / J_{1}\right) \otimes \delta(P),\left(\pi_{2} \otimes \mathrm{id}\right)(x) \in\left(C / J_{2}\right) \otimes \mathbb{C} \otimes H\right\}
\end{aligned}
$$

the equivariant fusion algebra of $P$ and $H$ over $C$. When $C:=C([0,1])$ and $\pi_{1}:=\mathrm{ev}_{1}, \pi_{2}:=\mathrm{ev}_{0}$, we call $P \circledast_{\pi}^{\delta} H$ the equivariant join algebra of $P$ and $H$, and denote it by $P \circledast{ }^{\delta} H$.

Lemma 5.2. The coaction $\mathrm{id} \otimes \mathrm{id} \otimes \Delta: C \otimes P \otimes H \rightarrow C \otimes P \otimes H \otimes H$ restricts and corestricts to $\Delta_{P \circledast_{\pi}^{\delta} H}: P \circledast_{\pi}^{\delta} H \rightarrow\left(P \circledast_{\pi}^{\delta} H\right) \otimes H$ making $P \circledast_{\pi}^{\delta} H$ a right $H$-comodule algebra.

Proof. Note first that for $i \in\{1,2\}$, we have

$$
\left(\left(\pi_{i} \otimes \mathrm{id} \otimes \mathrm{id}\right) \circ(\mathrm{id} \otimes \mathrm{id} \otimes \Delta)\right)\left(\sum_{j} f_{j} \otimes p_{j} \otimes h_{j}\right)=(\mathrm{id} \otimes \mathrm{id} \otimes \Delta)\left(\sum_{j} \pi_{i}\left(f_{j}\right) \otimes p_{j} \otimes h_{j}\right) .
$$

Assume now that $\sum_{j} f_{j} \otimes p_{j} \otimes h_{j} \in P \circledast_{\pi}^{\delta} H$. Then the above tensor belongs to

$$
\left(C / J_{1}\right) \otimes((\mathrm{id} \otimes \Delta)(\delta(P)))=\left(C / J_{1}\right) \otimes((\delta \otimes \mathrm{id})(\delta(P))) \subseteq\left(C / J_{1}\right) \otimes \delta(P) \otimes H
$$

for $i=1$ and to $\left(C / J_{2}\right) \otimes \mathbb{C} \otimes H \otimes H$ for $i=2$.

The main result of this paper is:

Theorem 5.3. Let $C$ be a unital $C^{*}$-algebra equipped with two $C^{*}$-ideals $J_{1}$ and $J_{2}$ such that the direct sum $\pi_{1} \oplus \pi_{2}: C \rightarrow\left(C / J_{1}\right) \oplus\left(C / J_{2}\right)$ of the canonical surjections $\pi_{i}: C \rightarrow C / J_{i}, i \in\{1,2\}$, is surjective. Then, for any principal right $H$-comodule algebra $P$, the equivariant fusion right $H$-comodule algebra $P \circledast_{\pi}^{\delta} H$ is principal.

Proof. Note first that, due to the surjectivity of $\pi_{1} \oplus \pi_{2}$, we can choose $x \in C$ such that

$$
\left(\pi_{1} \oplus \pi_{2}\right)(x)=(1,0) .
$$

Since $\{0,1\} \subseteq \operatorname{spec}\left(x^{*} x\right)$, we can take a continuous function $f: \operatorname{spec}\left(x^{*} x\right) \rightarrow[0,1]$ such that $f(0)=0$ and $f(1)=1$, and define $t:=f\left(x^{*} x\right) \in C$. Then there exist elements $\sqrt{t}, \sqrt{1-t} \in C$ enjoying the properties $\pi_{2}(\sqrt{t})=0=\pi_{1}(\sqrt{1-t})$.

Next, let $\ell: H \rightarrow P \otimes P$ be a strong connection on $P$. Then the bicolinearity of $\ell$ implies that the linear map

$$
\begin{aligned}
\tilde{\ell}: H & \longrightarrow(C \otimes P \otimes H) \otimes(C \otimes P \otimes H), \\
\tilde{\ell}(h):= & \sqrt{t} \otimes \ell\left(h_{(2)}\right)\langle 1\rangle \otimes S\left(h_{(1)}\right) \otimes \sqrt{t} \otimes \ell\left(h_{(2)}\right)^{\langle 2\rangle} \otimes h_{(3)} \\
& +\sqrt{1-t} \otimes 1 \otimes S\left(h_{(1)}\right) \otimes \sqrt{1-t} \otimes 1 \otimes h_{(2)},
\end{aligned}
$$


corestricts to $\left(P \circledast_{\pi}^{\delta} H\right) \otimes\left(P \circledast_{\pi}^{\delta} H\right)$. Indeed,

$$
\begin{aligned}
& \left(\pi_{1} \otimes \mathrm{id} \otimes \mathrm{id}\right) \otimes(\mathrm{id} \otimes \mathrm{id} \otimes \mathrm{id})(\widetilde{\ell}(h)) \\
& \quad=\left([1] \otimes \delta\left(\ell\left(h_{(1)}\right)^{\langle 1\rangle}\right)\right) \otimes\left(\sqrt{t} \otimes \ell\left(h_{(1)}\right)^{\langle 2\rangle} \otimes h_{(2)}\right) \in\left(C / J_{1} \otimes \delta(P)\right) \otimes(C \otimes P \otimes H), \\
& \left(\pi_{2} \otimes \mathrm{id} \otimes \mathrm{id}\right) \otimes(\mathrm{id} \otimes \mathrm{id} \otimes \mathrm{id})(\widetilde{\ell}(h)) \\
& \quad=\left([1] \otimes 1 \otimes S\left(h_{(1)}\right) \otimes\left(\sqrt{1-t} \otimes 1 \otimes h_{(2)}\right) \in\left(C / J_{2} \otimes \mathbb{C} \otimes H\right) \otimes(C \otimes P \otimes H) .\right.
\end{aligned}
$$

Much in the same way,

$$
\begin{aligned}
& (\mathrm{id} \otimes \mathrm{id} \otimes \mathrm{id}) \otimes\left(\pi_{1} \otimes \mathrm{id} \otimes \mathrm{id}\right)(\widetilde{\ell}(h)) \in(C \otimes P \otimes H) \otimes\left(C / J_{1} \otimes \delta(P)\right), \\
& (\mathrm{id} \otimes \mathrm{id} \otimes \mathrm{id}) \otimes\left(\pi_{2} \otimes \mathrm{id} \otimes \mathrm{id}\right)(\widetilde{\ell}(h)) \in(C \otimes P \otimes H) \otimes\left(C / J_{2} \otimes \mathbb{C} \otimes H\right) .
\end{aligned}
$$

The bicolinearity of the thus corestricted $\tilde{\ell}$ is evident. Finally, taking advantage of the right colinearity and the splitting property of $\ell$, we check that

$$
\begin{aligned}
\widetilde{\operatorname{can}_{P \circledast \pi}^{\delta} H}(\tilde{\ell}(h))= & t \otimes \ell\left(h_{(2)}\right)^{\langle 1\rangle} \ell\left(h_{(2)}\right)^{\langle 2\rangle} \otimes S\left(h_{(1)}\right) h_{(3)} \otimes h_{(4)} \\
& +(1-t) \otimes 1 \otimes S\left(h_{(1)}\right) h_{(2)} \otimes h_{(3)} \\
= & t \otimes \ell\left(h_{(2)}\right)^{\langle 1\rangle} \ell\left(h_{(2)}\right)^{\langle 2\rangle}(0) \otimes S\left(h_{(1)}\right) \ell\left(h_{(2)}\right)_{(1)}^{\langle 2\rangle} \otimes h_{(3)}+(1-t) \otimes 1 \otimes 1 \otimes h \\
= & t \otimes 1 \otimes S\left(h_{(1)}\right) h_{(2)} \otimes h_{(3)}+(1-t) \otimes 1 \otimes 1 \otimes h \\
= & 1 \otimes 1 \otimes 1 \otimes h .
\end{aligned}
$$

Now the claim follows from Lemma 3.3.

Observe that in the special case of the join construction, we can take $t$ to be the inclusion map $[0,1] \rightarrow \mathbb{C}$. Moreover, as explained in the next section, the equivariant join comodule algebra $P \circledast^{\delta} H$ becomes piecewise trivial, and Theorem 5.3 follows from [7, Lemma 3.2]. However, even in this special case of the equivariant join comodule algebra, our non-unital strongconnection formula is differently constructed than the complicated strong-connection formula in [7, Lemma 3.2]. This might be very important for index pairing computations involving concrete strong-connection formulas. Finally, let us remark that a fully fledged $C^{*}$-algebraic approach to Theorem 5.3 can be found in [5].

Example 5.4. In particular, taking $P=H$ to be the Hopf algebra of $\mathrm{SU}_{q}(2)$, we can conclude the principality of Pflaum's noncommutative instanton bundle [10]. The fact that this bundle is not trivializable at the $C^{*}$-algebraic level was proved in [1] using [4]. In the classical setting, except for the trivial group, all joins $G * G$, where $G$ is a compact Hausdorff group, are non-trivial as principal $G$-bundles. We conjecture the same is true for all compact quantum groups [13].

\section{$6 \quad$ Piecewise structure}

This section is an adjustment of [7, Section 5.1] to the setting of equivariant join construction. It is important to bear in mind that we cannot expect to have such a piecewise (pullback) structure in the general case of equivariant fusion comodule algebra.

Let $P$ be a principal right $H$-comodule algebra with a coaction $\delta$. First we define the following algebras

$$
\begin{aligned}
& \left.P_{1}:=\left\{x \in C([0,1]) \otimes P \otimes H \mid\left(\mathrm{ev}_{0} \otimes \mathrm{id} \otimes \mathrm{id}\right)(x) \in \mathbb{C} \otimes H\right)\right\}, \\
& P_{2}:=\left\{x \in C([0,1]) \otimes P \otimes H \mid\left(\mathrm{ev}_{1} \otimes \mathrm{id} \otimes \mathrm{id}\right)(x) \in \delta(P)\right\} .
\end{aligned}
$$


The $P_{i}$ 's become $H$-comodule algebras for the coactions obtained by the restrictions and corestrictions of id $\otimes$ id $\otimes \Delta$, and the subalgebras of $H$-coaction invariants are respectively

$$
\begin{aligned}
& B_{1}:=\left\{y \in C([0,1]) \otimes P \mid\left(\mathrm{ev}_{0} \otimes \mathrm{id}\right)(y) \in \mathbb{C}\right\}, \\
& B_{2}:=\left\{y \in C([0,1]) \otimes P \mid\left(\mathrm{ev}_{1} \otimes \mathrm{id}\right)(y) \in P^{\mathrm{co} H}\right\} .
\end{aligned}
$$

Now one can identify $P$ with the pullback comodule algebra

$$
\left\{(p, q) \in P_{1} \oplus P_{2} \mid\left(\mathrm{ev}_{1} \otimes \mathrm{id}\right)(p)=\left(\mathrm{ev}_{0} \otimes \mathrm{id}\right)(q)\right\}
$$

of the $P_{i}$ 's along the right $H$-colinear algebra homomorphisms

$$
\left(\mathrm{ev}_{1} \otimes \mathrm{id}\right): P_{1} \longrightarrow P \otimes H, \quad\left(\mathrm{ev}_{0} \otimes \mathrm{id}\right): P_{2} \longrightarrow P \otimes H .
$$

\section{Acknowledgements}

All authors are grateful to Piotr M. Soltan and Karen R. Strung for references concerning the minimal tensor product and the Jiang-Su $C^{*}$-algebra respectively. Ludwik Dąbrowski and Piotr M. Hajac were partially supported by PRIN 2010-11 grant "Operator Algebras, Noncommutative Geometry and Applications" and NCN grant 2011/01/B/ST1/06474, respectively. Tom Hadfield was financed via the EU Transfer of Knowledge contract MKTD-CT-2004-509794. Also, Piotr M. Hajac is very thankful to SISSA for its hospitality.

\section{References}

[1] Baum P.F., Dạbrowski L., Hajac P.M., Noncommutative Borsuk-Ulam-type conjectures, Banach Center Publ., Polish Acad. Sci. Inst. Math., Warsaw, to appear, arXiv:1502.05756.

[2] Baum P.F., De Commer K., Hajac P.M., Free actions of compact quantum group on unital $C^{*}$-algebras, arXiv:1304.2812.

[3] Brzeziński T., Hajac P.M., The Chern-Galois character, C. R. Math. Acad. Sci. Paris 338 (2004), 113-116, math.KT/0306436.

[4] Dąbrowski L., Hadfield T., Hajac P.M., Matthes R., Wagner E., Index pairings for pullbacks of $C^{*}$-algebras, in Operator Algebras and Quantum Groups, Banach Center Publ., Vol. 98, Polish Acad. Sci. Inst. Math., Warsaw, 2012, 67-84, math.QA/0702001.

[5] Da̧browski L., De Commer K., Hajac P.M., Wagner E., Noncommutative bordism of free actions of compact quantum groups on unital $C^{*}$-algebras, in preparation.

[6] Hajac P.M., Strong connections on quantum principal bundles, Comm. Math. Phys. 182 (1996), 579-617, hep-th/9406129.

[7] Hajac P.M., Krähmer U., Matthes R., Zieliński B., Piecewise principal comodule algebras, J. Noncommut. Geom. 5 (2011), 591-614, arXiv:0707.1344.

[8] Jiang X., Su H., On a simple unital projectionless $C^{*}$-algebra, Amer. J. Math. 121 (1999), 359-413.

[9] Milnor J., Construction of universal bundles. II, Ann. of Math. 63 (1956), 430-436.

[10] Pflaum M.J., Quantum groups on fibre bundles, Comm. Math. Phys. 166 (1994), 279-315, hep-th/9401085.

[11] Takesaki M., Theory of operator algebras. I, Springer-Verlag, New York - Heidelberg, 1979.

[12] Wassermann S., Exact $C^{*}$-algebras and related topics, Lecture Notes Series, Vol. 19, Seoul National University, Research Institute of Mathematics, Global Analysis Research Center, Seoul, 1994.

[13] Woronowicz S.L., Compact quantum groups, in Symétries Quantiques (Les Houches, 1995), North-Holland, Amsterdam, 1998, 845-884. 